ANNALES

POLONICI MATHEMATICI

XLIX (1988)

\title{
Exactness of expanding mappings
}

\author{
by Jolanta Socala (Katowice)
}

\begin{abstract}
A new sufficient condition for exactness of piecewise expanding mappings on the $d$-dimensional cube $[0,1]^{d}$ is proved. It generalizes the results of $[6]$ concerning the piecewise expanding mappings on the unit interval.

1. Introduction. The purpose of this paper is to establish a sufficient condition for the exactness of piecewise expanding mappings on the $d$ dimensional cube. In the one-dimensional case, this problem is almost completely solved. The solution follows from the results of Rényi [10]. Rochlin [11], Lasota and Yorke [7], and Lasola and Mackey [6]. In the $d$ dimensional case, this problem has been investigated in [1], [5] and [13]. In particular, K. Krzyżewski and W. Szlenk proved the exactness for expanding mappings of compact manifolds under assumption $T(A)=A$. G. Pianigiani and J. A. Yorke obtained a somewhat analogous result for expanding mappings in the case $A \subset T(A)$. In the present paper we assume $T(A) \subset A$.

We shall study our problem from a statistical point of view as transformations of probability densities. This leads immediately to the idea of considering the properties of the corresponding Frobenius- Perron operators. The proof of our theorem is based on idea due to [6]. Section 2 of the paper contains some preliminary notation. The main result is proved in Section 3.
\end{abstract}

2. Preliminaries. Let $(X, \Sigma, m)$ be a $\sigma$-finite measure space and let $S: X$ $\rightarrow X$ be a given transformation. In what follows, we shall assume that $S$ is doubly measurable which means that $S(A) \in \Sigma$ and $S^{-1}(A) \in \Sigma$ for $A \in \Sigma$. We shall also assume that $S$ is non-singular, i.e., $m(A)=0$ implies $m\left(S^{-1}(A)\right)=0$. We shall deal with the space $L^{1}=L^{1}(X, \Sigma, m)$ and the norm $\|\cdot\|=\|\cdot\|_{L^{1}}$. By $D=D(X, \Sigma, m)$ we shall denote the set of all (normalized) densities on $X$, that is,

$$
D=\left\{f \in L^{1}: f \geqslant 0 \text { and }\|f\|=1\right\} .
$$

For any density $g$ we shall denote by $m_{g}$ the measure

$$
m_{y}(A)=\int_{A} g d m \quad \text { for } A \in \Sigma .
$$


An important role in our considerations is played by the FrobeniusPerron operator. For a given $S$, we detine the Frobenius- Perron operator $P$ corresponding to $S$ by

$$
\text { iffdm= } \prod_{s} f \mathrm{fdm} \text { for } A \in \Sigma \text { and } f \in L^{1}
$$

From (1) it can be seen that $P$ is linear and preserves the integral and is contractive in $L{ }^{\prime}$ that is. $\left.{ }_{1} P f\|\leqslant\| f \|\right)$.

To deline the exiciness of the system $(S, \mu)$ assume that $(X, \Sigma, \mu)$ is a normalized measure space and that $S: X \rightarrow X$ is a measure preserving transformation. If

$$
\lim _{n} \mu\left(S^{n}(A)\right)=1
$$

for every $A=\Sigma$ with $m(A)>0$, then the system $(S, \mu)$ is called exact.

We shall ase the following sufficient condition for exactness (see [8]).

Theorim 1. Let $(X, 2, m)$ he a $\sigma$-finite measure space and let $S: X \rightarrow X$ he doubly measurable and non-singular. Assume that there exists $h \in L^{1}, h \geqslant 0$, $\|h\|>0$, such : hat

$$
\lim _{n}\left\|\left(h-P^{n} f\right)^{+}\right\|=0 \quad \text { for } f \in D .
$$

Then theic is a unique density $g$ such that the system $\left(S, m_{y}\right)$ is exact.

In (2). : : denoles $\max (0.3)$. A non-negative function $h \in L^{1}$ satisfying (2) will be called il lower hound function for $P$.

We shall use some standard notions of the theory of differential inequalitics. A function $f:(a, b) \rightarrow R$ is called left lower semicontinuous if

$$
\liminf _{t \rightarrow 0} f(t-a) \geqslant f(t) \quad \text { for } t \in(a, b) \text {. }
$$

For any function $f:(a, b) \rightarrow R$ we may define its right lower derivative by selling

$$
\frac{d+f(i)}{d t}=\liminf _{\varepsilon>0} \frac{f(t+a)-f(t)}{\varepsilon} \text { for } t \in(a, b)
$$

It is well known (see [12]) that for every left lower semicontinuous function $f:(a, b) \rightarrow R$ the inequality

$$
\frac{d+f(t)}{d t} \leqslant K f(t) \quad \text { for } i \in(a, b)
$$

implies

$$
f(l) \leqslant f(s) e^{K(t-s)} \quad \text { for } t \in[s, b) .
$$


Now He are going to deline the expanding maps on $R^{\prime \prime}$. We sil thall a mallis $M$ is i-cexpansice if inf $:|M \cdot v|:|v|=1 ! \geqslant i$. Let $S: X \rightarrow R^{d}, X \subset R^{\prime \prime}$ be aC'function and let $D S(x)$ denote the Jacobian matrix for $S$. We call the mapping $S$ i-expansite if $D S(x)$ is a i-expansive matrix for all $x \in X$.

3. The main result. Denote by $m$ the Lebesgue measure on the unit cube $X=[0,1]^{d}$. We shall write $x=\left(x_{1}, \ldots, x_{d}\right)$ for $x \in X$. Let $X=\bigcup_{i=1}^{k} X_{i}$. where $X_{i}, i=1, \ldots k$, are closed cubes which can be written in the form

$$
X_{i}=: x \in X: a_{j}^{i} \leqslant \lambda_{j} \leqslant b_{j}^{i} \text { for } j=1, \ldots, d^{\prime} .
$$

Assume that $m\left(X_{i} \cap X_{j}\right)=0$ for $i \neq j$. Consider al secuence of mappings $S^{i}: X_{i} \rightarrow X, i=1, \ldots, k$, which satisfy the condition:s:

(i) $S^{i}$ is a $C^{2}$-diffeomorphism onto its imaise

(ii) $\hat{\imath} T_{j}^{i} / \hat{\imath} x_{l} \geqslant 0$, des $D T^{i}>0$ for $j, l=1, \ldots$, whe whe $T^{i}=\left(S^{i}\right)^{-1}$ and $T^{i}$ $=\left(T_{1}^{i}, \ldots, T_{d}^{i}\right)$;

(iii) there exists $i>1$ such that $S^{i}$ is $i$-expansive:

(iv) if $x \in X_{i}$ and $x_{j} \neq a_{j}^{i}$ for a given $1 \leqslant j \leqslant d$, then $S_{j}^{i}(x) \neq 0$ :

(v) if $x \in Y_{i}=S^{i}\left(X_{i}\right)$ and $y_{j} \leqslant x_{j}$ for $j=1, \ldots$, d, then $y \in Y_{i}$.

We define the mapping $S: X \rightarrow X$ by the condition

(vi) $S(x)=S^{i}(x)$ for $x \in \operatorname{int} X_{i}, i=1, \ldots, k$.

THFOREM 2. If $S$ is given by formula (vi) and $S^{i}$ satisfy conditions (i) (v). then there exists a unique density $g$ such that the system $\left(S, m_{g}\right)$ is exact

Proof. Let $Q$ be the set of all $C^{1}$-functions $q: A \rightarrow X$ such that

$$
d q_{j} / d t \geqslant 0 \quad \text { for } j=1, \ldots, d \text { and } t \in \Delta \text {, }
$$

where $\Delta$ is a compact interval which depends in general upon $q$. Now let $D_{0}$ denote the subset of $D=D(X, \Sigma, m)$ consisting of all functions $f$ satisfying for every $q \in Q$ the following two conditions:

(C1) The function $f \circ q$ is left lower semicontinuous:

$$
\frac{d_{+}}{d t} \frac{f \circ q}{d t} \leqslant K_{f}\left|\frac{d q}{d t}\right| f \circ q
$$

where $|\cdot|$ stands for the norm in $R^{d}$ and the constant $K_{f}$ depends in general on $f$ but not on $q$. The proof will be given in two steps. First, we shall show that $P^{n} f \in D_{0}$ for $f \in D_{0}$ and that there exists a constant $K$ such that $K_{p^{n} f} \leqslant K$ for every $f \in D_{0}$ and for $n \geqslant n(f)$. Then in Step II we shall prove the existence of a constant $r$ such that for every $f \in D_{0}$

$$
\int_{B_{r}} P^{n} f d m \leqslant 1 / 2, \quad B_{r}=i, x \in X: x_{j} \leqslant r \text { for some } j_{1}^{?}
$$


for sufficiently large $n$. This allows to construct a non-trivial (different from zero) lower bound function for $P$.

Step I. Let $f \in D_{0}$ and $q \in Q$. A simple computation shows that the operator $P$ can be written in the form

$$
P f(x)=\sum_{i=1}^{k} \mathbf{1}_{Y_{i}}(x) f \circ T^{i}(x) \operatorname{det} D T^{i}(x)
$$

Hence

$$
P f \circ q(t)=\sum_{i=1}^{k} \mathbf{1}_{Y_{i}} \circ q(t) f \circ T^{i} \circ q(t) \operatorname{det} D T^{i} \circ q(t) \quad \text { for } t \in \mathcal{A} .
$$

From conditions (v) and (ii) it follows that

$$
\mathbf{1}_{Y_{i}} \circ q=\mathbf{1}_{\left[0 . h_{i}\right]} \quad \text { and }\left.\quad T^{i} \circ q\right|_{\left[0 . h_{i}\right]} \in Q
$$

where $b_{i}=\sup , t: q(t) \in Y_{i}$, The function $P f \circ q$ as calculated from equation (5) is left lower semicontinuous. Differentiation of (5) gives

$$
\begin{aligned}
& \frac{d_{+} P f \circ q}{d t} \\
& =\sum_{i=1}^{k} \mathbf{1}_{Y_{i}} \circ q \frac{d+f \circ T^{i} \circ q}{d t} \operatorname{det} D T^{i} \circ q+\sum_{i=1}^{k} \mathbf{1}_{Y_{i}} \circ q \frac{d\left(\operatorname{det} D T^{i} \circ q\right)}{d t} f \circ T^{i} \circ q .
\end{aligned}
$$

By conditions (C2) and (iii) we obtain

$$
\frac{d+f \circ T^{i} \circ q}{d t} \leqslant K_{f}\left|\frac{d T^{i} \circ q}{d t}\right| f \circ T^{i} \circ q \leqslant \beta K_{f}\left|\frac{d q}{d t}\right| f \circ T^{i} \circ q
$$

where $\beta=1 / \lambda$. Since $Y_{i}$ are compact, there exists a constant $L$ such that

$$
\frac{\hat{\jmath} \operatorname{det}}{\imath} \frac{D T^{i}}{x_{j}} \leqslant L \operatorname{det} D T^{i} \quad \text { for } i=1, \ldots, k ; j=1, \ldots, d
$$

so, as a consequence,

$$
\frac{d\left(\operatorname{det} D T^{i} \circ q\right)}{d t} \leqslant L \operatorname{det} D T^{i} \circ q \sum_{j=1}^{d} \frac{d q_{j}}{d t} \leqslant d L \operatorname{det} D T^{i} \circ q\left|\frac{d q}{d t}\right| .
$$

Thus, finally

$$
\frac{d_{+} P f \circ q}{d t} \leqslant\left(\beta K_{f}+d L\right)\left|\frac{d q}{d t}\right| \operatorname{Pf} \circ q
$$

and $P f \in D_{0}$ with a constant $K_{P f} \leqslant \beta K_{f}+d L$. An induction argument shows 
that $P^{n} f \in D_{0}$ and

$$
K_{P^{n_{f}}} \leqslant \beta^{n} K_{f}+d L /(1-\beta) \leqslant K
$$

for $n$ sufficiently large and $K=1+d L /(1-\beta)$.

Before passing to the Step II we shall proof three simple lemmas.

Lemma 1. Let $f \in D_{0}$ and $K_{f} \leqslant K$. If $x_{j} \leqslant y_{j}$ for $j=1, \ldots$, $d$, then $f(y) \leqslant M f(x)$, where $M=e^{k d}$.

Proof. Define $q: I \rightarrow X$ by the formula $q(t)=t y+(1-t) x$. Evidently $q \in Q$ and $|d q / d t| \leqslant d$. By the definition of $D_{0}$

$$
d_{+} f \circ q / d t \leqslant K d f \circ q
$$

and the function $f \circ q$ is left lower semicontinuous. From (3) it follows $f(y) \leqslant M f(x)$.

Lemma 2. Assume that $f \in D_{0}$ and $K_{f} \leqslant K$. Let $c, r$ be positive real numbers such that $c+r \leqslant 1$. Then

$$
\int_{c_{j}} f d m \leqslant r M / c \quad \text { where } C_{j}=\left\{x \in X: c \leqslant x_{j} \leqslant c+r\right\} .
$$

Proof. Let $B_{j}=\left\{x \in X: x_{j} \leqslant c\right\}$ and let

$$
F_{j}(x)=\left(x_{1}, \ldots, x_{j-1}, c+x_{j} r / c, x_{j+1}, \ldots, x_{d}\right)
$$

for $x \in B_{j}$. Then det $D F_{j}=r / c$, and consequently, from Lemma 1 it follows that

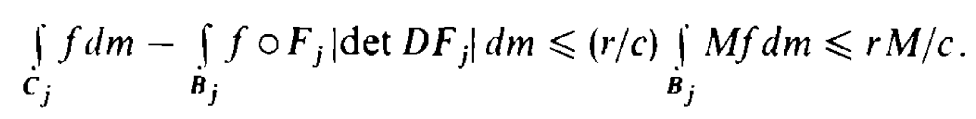

Lemma 3. Let $j(1 \leqslant j \leqslant d)$ be a given number and let $r$ be a positive real number. Then

$$
T^{i}\left(K_{r} \cap Y_{i}\right) \subset K_{\beta r}^{i} \quad \text { for } i=1, \ldots, k
$$

where

$$
K_{r}=\left\{x \in X: x_{j} \leqslant r_{\}}, \quad K_{\beta r}^{i}=\left\{x \in X_{i}: x_{j} \leqslant a_{j}^{i}+\beta r\right\} .\right.
$$

Proof. First we shall show that if $x, y \in Y_{i}$ for a certain $i$, then $\left|T^{i} x-T^{i} y\right| \leqslant \beta|x-y|$. Define $q: I \rightarrow X$ by the formula $q(t)=t y+(1-t) x$. Using condition (iii) we may write

$$
\left|T^{i} x-T^{i} y\right| \leqslant \int_{0}^{1}\left|\frac{d T^{i} \circ q}{d t}\right| d t \leqslant \beta \int_{0}^{1}\left|\frac{d q}{d t}\right| d t=\beta|x-y| .
$$

For a given point $x \in T^{i} K_{r}$ and for $y=S^{i} x$ we have $y_{j} \leqslant r$. Set $z$ $=\left(y_{1}, \ldots, y_{j-1}, 0, y_{j+1}, \ldots, y_{d}\right)$. From (iv) and (v) it follows that $z \in Y_{i}$ and 
$T_{j}^{i} z=a_{j}^{i}$. Thus, since

$$
x_{j}-a_{j}^{i} \leqslant\left|x-T^{i} z\right| \leqslant \beta|y-z| \leqslant \beta r,
$$

we have $x \in K_{\beta r}^{i}$.

Step II. Define $B_{r}$ as in formula (4). For a given $j(1 \leqslant j \leqslant d)$, set

$$
I=\left\{i: a_{j}^{i}=0\right\}, \quad J=\left\{i: a_{j}^{i} \neq 0\right\} \quad \text { and } \quad b=\min \left\{a_{j}^{i}: i \in J\right\} .
$$

Let $K_{r}, K_{\beta r}^{i}$ be as in Lemma 3. If $f \in D_{0}$, then there exists $N$ such that $K_{p^{n} j} \leqslant K$ for $n \geqslant N$. Since $m\left(X_{i} \cap X_{j}\right)=0$ for $i \neq j$, it follows that $m\left(K_{\beta \mathbf{r}}^{i} \cap K_{\beta r}^{j}\right)=0$ for $i \neq j$. From Lemma 3 and Lemma 2 we have

$$
\begin{aligned}
\int_{\kappa_{r}} P^{N+1} f d m & =\sum_{i=1}^{k} \int_{T^{i}\left(K_{r}\right)} P^{N} f d m \leqslant \sum_{i=1}^{k} \int_{\substack{K_{\beta r} \\
i}} P^{N} f d m \\
& \leqslant \sum_{i \in I} \int_{\substack{\kappa_{\beta r} \\
i}} P^{N} f d m+\sum_{i \in J} \int_{\substack{i \\
\kappa_{\beta r}}} P^{N} f d m \leqslant \underset{\kappa_{\beta r}}{\int} P^{N} f d m+k \beta r M / b .
\end{aligned}
$$

Again, using a simple induction argument it follows that

$$
\int_{K_{\boldsymbol{r}}} P^{N+n} f d m \leqslant \int_{\boldsymbol{K}^{n_{r}}} P^{N} f d m+k \beta r M / b(1-\beta) \leqslant 1 / 2 d
$$

and consequently

$$
\int_{B_{r}} P^{N+n} f d m \leqslant 1 / 2
$$

for $r<b(1-\beta) / 2 d k \beta M$ and $n$ sufficiently large.

We are going to show that $h=(1 / 2 M) \mathbf{1}_{G}$ with

$$
G=\left\{x \in X: x_{j} \leqslant r \text { for every } j=1, \ldots, d\right\}
$$

is a lower bound function for $P$. Let $f \in D_{0}$ and

$$
K_{p^{n_{f}}} \leqslant K \quad \text { and } \quad \int_{\boldsymbol{B}_{\boldsymbol{r}}} P^{n} f d m \leqslant 1 / 2 \quad \text { for } n \geqslant N .
$$

From Lemma 1 for $x \in G$ we have

$$
1 / 2 \leqslant \int_{X-B_{\boldsymbol{r}}} P^{n} f d m \leqslant \int_{X-B_{\mathbf{r}}} M P^{n} f(x) d m \leqslant M P^{n} f(x)
$$

and $P^{n} f(x) \geqslant 1 / 2 M$. Since the set $D_{0}$ is dense in $D$, this completes the proof.

Remark. The existence of absolutely continuous invariant measure for a certain class of piecewise differentiable mappings on the $d$-dimensional cube 
has been proved in [2]-[4]. The transformation

$$
S(x, y)= \begin{cases}(4 x-4 x y, 4 y) & \text { for } 0 \leqslant x, y \leqslant 1 / 4, \\ (4 x, 4 y)(\bmod 1) & \text { elsewhere }\end{cases}
$$

on the unit square $I^{2}$ satisfies the assumptions of our Theorem 2 and it is not of the form considered in the mentioned papers.

\section{References}

[1] A. Avez, Propriétés ergodiques des endomorphismes dilatants des variétés compactes, C. R. Acad. Sci. Paris Sér A-B 266 (1968), A610-A612.

[2] H. Haller, Rectangle exchange transformations, Monatsh. Math. 91 (1981), 215-232.

[3] M. Jabloński, On invariant measures for piecewise convex transformations, Ann. Polon. Math. 32 (1976), 207-214.

[4] -, Invariant measures for piecewise $C^{2}$-transformations, ibidem 43 (1983), 185-195.

[5] K. Krzyżewski and W. Szlenk, On invariant measures for expanding differentiable mappings, Studia Math. 33 (1969), 83-92.

[6] A. Lasola and M. C. Mackey, Probabilistic Properties of Deterministic Systems, Cambridge University Press. Cambridge 1986.

[7] A. Lasola and J. A. Yorke, On the existence of invariant measures for piecewise monotonic transformations, Trans. Amer. Math. Soc. 186 (1973), 481-488.

[8] -, -, Exact dynamical systems and the Frobenius-Perron operator, ibidem 273 (1982), $375 \cdot 384$

[9] G. Pianigiani and J. A. Yorke, Expanding maps on sets which are almost intarianr: decay and chars. ibidem 252 (1979), 351-365.

[10] A. Rényi, Representation for real numbers and their ergodic properties, Acta Math. Acad. Sci. Hungar. 8 (1957), 477-493.

[11] V. A. Rochlin, Exact endomorphisms of Lebesgue spaces, Izv. Akad. Nauk SSSR Ser. Mat. 25 (1971), 499-530; Amer. Math. Soc. Transl. (2) 39 (1964), 1-36.

[12] J. A. Walker, Dynamical Systems and Evolution Equations, Plenum Press, New York 1980.

[13] M. S. Waterman, Some ergodic properties of multidimensional F-expansions, Z. Wahrscheinlichkeitstheorie und Verw. Gebiete 16 (1970), 77-103. 